\title{
Methicillin Resistant Staphylococcus aureus Isolated from Wound Infections
}

\author{
Jyoti Shrestha ${ }^{1}$, Krishna Govinda Prajapati ${ }^{2}$, Om Prakash Panta ${ }^{1}$, Pramod Poudel ${ }^{1}$ \\ and Santosh Khanal ${ }^{*}$ \\ ${ }^{1}$ Department of Microbiology, National College, Kathmandu, Nepal \\ ${ }^{2}$ Department of Microbiology, B and B Hospital, Lalitpur, Nepal
}

\begin{abstract}
*Corresponding author: Santosh Khanal, Department of Microbiology, National College, Kathmandu, Nepal E-mail: santoshkhanal007@gmail.com
\end{abstract}

\begin{abstract}
Objectives: The aim of this study was to determine the prevalence of methicillin resistant Staphylococcus aureus (MRSA) and assess antibiotic resistance pattern of the isolates from wound infections.

Methods: A total of 706 wound specimens including pus and wound swab were processed in the laboratory of B and B Hospital, Lalitpur from May to October 2014. The specimens were cultured on blood agar and mannitol salt agar plates and incubated at $37^{\circ} \mathrm{C}$ for 24 hours. Antibiotic susceptibility test was performed by modified Kirby-Bauer disc diffusion method. Strains resistant to cefoxitin (30 mcg) with inhibition zone $\leq 21 \mathrm{~mm}$ were identified as MRSA.
\end{abstract}

Results: Out of 366 bacterial isolates, 90 (24.6\%) were S. aureus and among them 16.7\% were MRSA and $54.4 \%$ multi-drug resistant (MDR). All isolates were sensitive to vancomycin and most of the isolates were sensitive to cefoxitin (83.3\%). High rate of resistance was observed towards penicillin (98.9\%) and ampicillin (86.7\%). All MRSA isolates and 52.9\% of methicillin sensitive S. aureus (MSSA) were MDR.

Conclusion: MRSA incidence is increasing in the population, and therapeutic measures are few and accompanied by diverse side effects. It is noteworthy to state that vancomycin is still the first line drug although vancomycin-resistant strains have been reported.

Key words: Wound infection, antimicrobial resistance, MRSA, MDR

\section{INTRODUCTION}

Wound is a breach in the skin, which can lead to infections with the presence of replicating microorganisms with the discharge of pus (Dulon et al. 2011). Staphylococcus aureus has been recognized as an important cause of disease around the world ranging from relatively mild infections of the skin and soft tissue to life-threatening sepsis. The emergence of strains resistant to methicillin and other antimicrobial agents has become a major concern, especially in the hospital environment (Spagnolo et al. 2014).

Methicillin resistance is mediated by PBP-2a, a penicillin binding protein encoded by the mec $A$ gene that is located on a mobile genetic element called a Staphylococcal cassette chromosome (Mahasenan et al.

Date of Submission: August 7, 2018

Published Online: January 2019
2017). The relative ease of transfer of this genetic element explains the growing resistance to $\beta$-lactam antibiotics such as penicillin and its chemical derivatives as well as cephalosporins. MRSA is now endemic in both community and hospital environments (Sit et al. 2017).

MRSA strains have spread among hospitals and disseminated worldwide. The development of resistance to multiple antibiotics and control of disease transmissions by MRSA isolates in hospitals have been recognized as a major challenge (Chen and Huang 2014). The recent studies conducted in different parts of Nepal reported the rate of MRSA to be $21.1 \%$ (Khanal et al. 2018), 35.5\% (Adhikari et al. 2017) and 43.6\% (Raut et al. 2017). The knowledge on prevalence of MRSA and their current antimicrobial profile has become

Date of Acceptance: December 10, 2018

DOI: https:/ / doi.org/10.3126/tujm.v5i0.22297 
necessary in the selection of appropriate empirical treatment of these infections. This study aimed to determine the prevalence of MRSA in wound infections among the patients attending B and B hospital and their susceptibility pattern towards various antimicrobial agents.

\section{MATERIALS AND METHODS}

\section{Study site and population}

The research work was conducted at laboratory of Department of Microbiology, B and B Hospital, Gwarko, Lalitpur from May to October 2014. During this period, a total of 706 aspirated pus and wound swabs from male and female patients of age group 1-87 years was collected, properly labelled and transferred to laboratory for further processing.

\section{Isolation and identification of $S$. aureus}

The specimens were directly inoculated on blood agar and mannitol salt agar plates and incubated at $37^{\circ} \mathrm{C}$ for 24 hours. The isolates were identified by morphological appearance of the colonies, microscopic findings; biochemical properties like catalase production test and coagulase production test by slide and tube methods. The colonies with golden yellow pigmentation on mannitol salt adgar and cream colored hemolytic or non-hemolytic on blood agar; Gram-positive cocci in grape-like cluster in Gram staining and catalase and

Table 1: Bacterial growth pattern in wound samples

\begin{tabular}{llll}
\hline Specimens & Bacterial growth N (\%) & No growth N (\%) & Total sample \\
\hline Wound swab & $352(52.8)$ & $315(47.2)$ & $667(94.5)$ \\
Aspirated pus & $14(35.9)$ & $25(64.1)$ & $39(5.5)$ \\
\hline Total & $366(51.8)$ & $340(48.2)$ & $706(100)$ \\
\hline
\end{tabular}

Among 366 isolated different organisms, 90 (24.6\%) were identified as $S$. aureus. Klebsiella species was found to be most predominent Gram negative bacteria constituting 89 (24.3\%). Other most frequently isolated organisms were E. coli $(22.1 \%)$ and Enterococcus spp (11\%) (Table 2). coagulase tests positive were identified as $S$. aureus (Forbes et al. 2007).

\section{Antibiotic susceptibility testing}

Antibiotic susceptibility tests of all $S$. aureus isolates towards various antibiotics were performed by modified Kirby-Bauer disk diffusion method as recommended by Clinical Laboratory Standard Institute (CLSI 2018). In this study the antibiotics used were amoxicillin (AMX/10mcg), cefoxitin (CX/30mcg), ciprofloxacin (CIP/5mcg), cotrimoxazole (COT $/ 25 \mathrm{mcg})$, erythromycin (E/15mcg), gentamicin (GEN/10mcg), ofloxacin (OF/5mcg), penicillin (P/10mcg) and vancomycin (VA/30 mcg). Screening for methicillin resistance was performed by cefoxitin disc diffusion method and interpreted according to CLSI (2018). Isolates with diameter of zone of inhibition $(\mathrm{ZOI}) \geq 22 \mathrm{~mm}$ were identified as MSSA and isolates with ZOI $\leq$ $21 \mathrm{~mm}$ identified as MRSA. Isolates resistant to three or more classes of antibiotics were considered MDR

\section{RESULTS}

A total of 706 wound specimens included 39 aspirated pus and 667 wound swabs in which bacterial growth was observed in 366 wound specimens (Table 1). (Nair et al. 2013).

Table 2: Different bacterial isolates from wound infection

\begin{tabular}{lll}
\hline Organisms & Number of isolates & $\%$ \\
\hline Staphylococcus aureus & 90 & 24.6 \\
Klebsiella spp & 89 & 24.3 \\
E. coli & 81 & 22.1 \\
Enterococcus spp & 40 & 11 \\
Proteus spp & 35 & 9.6 \\
Pseudomonas spp & 21 & 5.7 \\
Citrobacter spp & 6 & 1.6 \\
Acinetobacter spp & 4 & 1.1 \\
\hline Total & 366 & 100 \\
\hline
\end{tabular}


Out of 706 patients under study, 470 (66.6\%) were inpatients and $236(33.4 \%)$ were outpatients. The culture positive cases in inpatients were $216(59 \%)$ and $150(41 \%)$ in outpatients (Table 3 ).

Table 3: Case wise distribution of patients

\begin{tabular}{lcc}
\hline Patient type & Specimen N (\%) & Culture positive N (\%) \\
\hline Inpatients & $470(66.6)$ & $216(59)$ \\
Outpatients & $236(33.4)$ & $150(41)$ \\
\hline Total & 706 & 366 \\
\hline
\end{tabular}

The higher numbers of isolates of $S$. aureus were recovered from the age group 31-40 years (20\%) isolates were from age group 71-80 years (10\%) and followed by age group 21-30 years (17.8\%). Least none were isolated from 80 years and above (Table 4).

Table 4: S. aureus isolates from different age groups of patients

\begin{tabular}{|c|c|c|c|}
\hline \multirow{2}{*}{ Age group (years) } & \multirow{2}{*}{ Total } & \multicolumn{2}{|c|}{ S. aureus } \\
\hline & & $\mathrm{N}$ & $\%$ \\
\hline$<10$ & 41 & 6 & 6.7 \\
\hline $11-20$ & 72 & 11 & 12.2 \\
\hline $21-30$ & 85 & 16 & 17.8 \\
\hline $31-40$ & 196 & 18 & 20 \\
\hline $41-50$ & 174 & 13 & 14.4 \\
\hline $51-60$ & 35 & 12 & 13.3 \\
\hline $61-70$ & 60 & 5 & 5.6 \\
\hline $71-80$ & 33 & 9 & 10 \\
\hline 80 above & 10 & 0 & 0 \\
\hline Total & 706 & 90 & 100 \\
\hline
\end{tabular}

Out of 366 culture positive cases, a total of 90 S. aureus were isolated. Among them, 61 isolates (67.8\%) were from male patients and $29(32.2 \%)$ were from female patients. The distribution of $S$. aureus was higher in males than in females and the result was statistically significant $(\mathrm{p}<0.05)$ (Table 5).

Table 5: Distribution of S. aureus according to the gender of patients

\begin{tabular}{|c|c|c|c|}
\hline \multirow{2}{*}{ Gender } & \multicolumn{2}{|c|}{ S. aureus } & \multirow{2}{*}{$P$-value } \\
\hline & $N$ & $\%$ & \\
\hline Male & 61 & 67.8 & \multirow{3}{*}{0.005} \\
\hline Female & 29 & 32.2 & \\
\hline Total & 90 & 100 & \\
\hline
\end{tabular}

S. aureus isolated were tested with different antibiotics by using modified Kirby-Bauer disc diffusion method. Antibiotic susceptibility pattern of $S$. aureus isolates showed that the high proportion of isolates were resistant to penicillin $(\mathrm{n}=89,98.9 \%)$ and amoxicillin $(n=78,86.7 \%)$. All the isolates of $S$. aureus were susceptible to vancomycin and most of the isolates were susceptible to cefoxitin $(\mathrm{n}=75,83.3 \%)$ and gentamicin ( $n=48,53.3 \%$ ). The prevalence of MRSA was found to be $16.7 \%$ as shown by resistance with cefoxitin (Table 6). 
Table 6: Antibiotic susceptibility pattern of $S$. aureus $(\mathbf{N}=90)$

\begin{tabular}{lccc}
\hline Antibiotics & Sensitive N (\%) & Intermediate N (\%) & Resistant N (\%) \\
\hline Amoxicillin & $10(11.1)$ & $2(2.2)$ & $78(86.7)$ \\
Penicillin & $1(1.1)$ & 0 & $89(98.9)$ \\
Gentamicin & $48(53.3)$ & $8(8.9)$ & $34(37.8)$ \\
Cotrimoxazole & $41(45.6)$ & $27(30)$ & $22(24.4)$ \\
Cefoxitin & $75(83.3)$ & 0 & $15(16.7)$ \\
Erythromycin & $12(13.3)$ & $29(32.2)$ & $49(54.4)$ \\
Vancomycin & $90(100)$ & 0 & 0 \\
Ofloxacin & $30(33.3)$ & $9(10)$ & $51(56.7)$ \\
Ciprofloxacin & $30(33.3)$ & $7(7.8)$ & $53(58.9)$ \\
\hline
\end{tabular}

Among 90 S. aureus isolates, 49 (54.4\%) were found to were MDR (Table 7). be MDR. All 15 MRSA isolates and 34 (45.3\%) MSSA

Table 7: MDR pattern of $S$. aureus

\begin{tabular}{lccc}
\hline Drug resistance & MRSA N (\%) & MSSA N (\%) & Total S. aureus N (\%) \\
\hline MDR & $15(30.6)$ & $34(69.4)$ & $49(54.4 \%)$ \\
Non-MDR & 0 & $41(100)$ & $41(45.6 \%)$ \\
\hline Total & $15(16.7)$ & $75(83.3)$ & $90(100 \%)$ \\
\hline
\end{tabular}

\section{DISCUSSION}

MRSA has emerged as a serious public health problem globally. Because of the ability of Staphylococci to acquire antimicrobial resistance over time, MRSA has been and will continue to be a problem in the future. Today, most of the MRSA are multi-drug resistant thus causing a clinical problem as antibiotic treatment becomes useless. As such, this study was undertaken to determine the prevalence of $S$. aureus and MRSA, along with their antibiotic susceptibility patterns.

Out of 706 specimens, 366 were culture positive cases and S. aureus $(24.6 \%)$ was found to be predominant bacteria causing wound infection. Pandey et al. (2012) and Hussain et al. (2005) reported similar results with bacterial growth of $26.1 \%$ and $20 \%$ respectively. This suggested that $S$. aureus is the constantly isolated pathogen in hospital settings and regular intervention is required for the control of infection caused by this organism.

The present study showed that male $(67.8 \%)$ had a higher infection rate of wounds than females, which was statistically significant $(\mathrm{p}<0.05)$ and similar result was found in a study carried out by Mama et al. (2014). Some other studies showed statistically insignificant results in the distribution of $S$. aureus between males and females (Adhikari et al. 2017; Khanal et al. 2018). The number of wound specimens was highest in 31-40 years of age group with higher incidence of $S$. aureus infection (20\%). This might be explained by the fact that this group of population is mainly involved in occupations such as farming, construction works, transportation and industry works where the likely exposure to trauma is common.

Among 470 samples from inpatients, 216 culture positive results were observed and 150 positive cases were observed from 236 samples of outpatients. The prevalence of $S$. aureus was higher in outpatients $(33.3 \%)$ as compared to inpatients $(18.5 \%)$. The result was not in the agreement with the study done by Bhatta et al. (2014) who have reported higher prevalence of $S$. aureus in hospital setting accounting $54 \%$ as compared to outpatients $(46 \%)$.

S. aureus isolated in this study in overall showed higher rate of sensitivity towards cefoxitin (83.3\%) followed by gentamicin $(53.3 \%)$ and cotrimoxazole $(45.6 \%)$ whereas higher rate of resistance was observed towards penicillin (98.9\%) and amoxicillin (86.7\%) followed by ciprofloxacin $(58.9 \%)$ and erythromycin $(54.4 \%)$. In this study, most of $S$. aureus isolates were resistant towards $\beta$-lactam antibiotics making them the least effective drugs. The high resistance to penicillin and total susceptibility to vancomycin is commonly noted for $S$. aureus isolated at different hospitals worldwide (Adhikari et al. 2017). Bacterial 
resistance to $\beta$-lactam antibiotics is primarily due to the production of $\beta$-lactamase that opens its $\beta$-lactam ring rendering them to deactivate and also its penicillin binding protein (PBP2a) (Richmond 2000). None of the isolates were resistant to vancomycin as this antibiotic has unique mode of action to bacteria. It acts by inhibiting the second stage of cell wall synthesis of various susceptible bacteria altering bacterial cell wall membrane permeability and RNA synthesis (Rijal et al. 2008). Knowledge about MRSA and carrier status needs to be raised among the health staffs of the hospital and control measures need to be implemented consistently in order to reduce the burden of MRSA infection in the hospital environment (Holmes et al. 2005).

Among 90 isolates of S. aureus 49 isolates (54.4\%) were found to be MDR. Similar studies by Banjara (2002), Rajbhandari et al. (2003) and Surucuoqlu et al. (2005) reported 40\%,54.9\% and 31\% MDR S. aureus respectively from wound sample. The overuse of antibiotics clearly drives the evolution of resistance. In bacteria, antibiotic resistance occurs due to horizontal gene transfer among different species of bacteria and spontaneously through mutation. Antibiotics remove drug-sensitive competitors, leaving resistant bacteria behind to reproduce as a result of natural selection. Despite warnings regarding overuse, antibiotics are overprescribed worldwide (Read and Woods 2014).

The rapid emergence of resistant bacteria is occurring worldwide, endangering the efficacy of antibiotics, which have transformed medicine and saved millions of lives (Golkar et al. 2014). The antibiotic resistance crisis has been attributed to the overuse and misuse of these medications, as well as a lack of new drug development by the pharmaceutical industry due to reduced economic incentives and challenging regulatory requirements (Spellberg and Gilbert 2014).

\section{CONCLUSION}

$S$. aureus was the most common bacteria causing wound infection and the prevalence of MRSA and MDR S. aureus was $16.7 \%$ and $54.4 \%$ respectively. Most of the isolates were sensitive towards and gentamicin and resistant towards penicillin and amoxicillin. Continuous surveillance on antimicrobial susceptibility of $S$. aureus is essential for the detection of emerging trends and the development of appropriate therapeutic strategies.

\section{ACKNOWLEDGEMENTS}

We are most grateful to the managing director of $B$ and B Hospital for giving us the clinical facilities and the necessary support. We would like to thank all the staff of the National College for their support and suggestions.

\section{CONFLICT OF INTEREST}

The authors declare no conflict of interest.

\section{REFERENCES}

Adhikari R, Pant ND, NeupaneS, Neupane M, Bhattarai R, Bhatta S, Chaudhary R and Lekhak B (2017). Detection of Methicillin Resistant Staphylococcus aureus and Determination of Minimum Inhibitory Concentration of Vancomycin for Staphylococcus aureus Isolated from Pus/Wound Swab Samples of the Patients Attending a Tertiary Care Hospital in Kathmandu, Nepal. Can J Infect Dis Med Microbiol Volume 2017, Article ID 2191532.

Banjara MR (2002). Study of air, water and wound Infection in different wards of TUTH. A dissertation submitted to the central department of Microbiology, TU, Kathmandu, Nepal. pp 4247.

Bhatt CP, Karki BMS, Baral B, Gautam S, Shah A and Chaudhary A (2014). Antibiotic susceptibility pattern of Staphylococcus aureus and methicillinresistant Staphylococcus aureus in a tertiary care hospital. Journal of Pathology of Nepal 4: 548 - 551.

Chen CJ and Huang YC (2014). New epidemiology of Staphylococcus aureus infection in Asia. Clin Microbiol Infect 20(7): 605-623.

CLSI, Wayne, PA, USA (2018). Performance standards for anitmicrobial susceptibility testing, $28^{\text {th }}$ informational supplement M100-S28.

Dulon M, Haamann F, Peters C, Schablon A and Nienhaus A (2011). MRSA prevalence in european healthcare settings : a review. BMC Infect Dis 11: 138.

Forbes BA, Sahm DF and Weissfeld AS (2007). Staphylococcus, MicrococcusandSimilarOrganisms. In Baily and Scott's Diagnostic Microbiology. $12^{\text {th }}$ ed. Mosby Inc: St. Louis, pp. 254-280.

Golkar Z, Bagazra O and Pace DG (2014). Bacteriophage therapy: a potential solution for the antibiotic resistance crisis. J Infect Dev Count 8(2): 129-136.

Holmes A, Ganner M, McGuane S, Pitt TL, Cookson BD and Kearns AM (2005). Staphylococcus aureus 
isolates carrying frequency, characterization and association with clinical disease. J Clin Microbiol 43(5): 2384-2390.

Hussain S, Shams R, Ahmad K, Perveen R and Riaz B (2005). Prevalence of methicillin Resistant Staphylococcus aureus (MRSA) in Surgical Site Infections in a Tertiary Care Hospital. Int J Path 3(2): 81-85.

Khanal LK, Adhikari RP and GuragainA (2018). Prevalence of Methicillin Resistant Staphylococcus aureus and Antibiotic Susceptibility Pattern in a Tertiary Hospital in Nepal. J Nepal Health Res Counc 16(39): 172-174.

Mahasenan KV, Molina R, Bouley R, Batuecas MT, Fisher JF, Hermoso JA, Chang M and Mobashery S (2017). Conformational Dynamics in PenicillinBinding Protein 2a of Methicillin-Resistant Staphylococcus aureus, Allosteric Communication Network and Enablement of Catalysis. J Am Chem Soc 139: 2102-2110.

Mama M, Abdissa A and Sewunet T (2014). Antimicrobial susceptibility pattern of isolates from wound infection and their sensitivity to alternative topical agents at Jimma University Specialized Hospital, South West, Ethiopia. Ann Clin Microbiol Antimicrob 14: 13:14.

Nair R, Hanson BM, Tundev O and Smith TC (2013). Antimicrobial resistantance and molecular epidemeology of Staphylococcus aureus from Ulanbattar, Mongolia. Peer J 1: e176.

Pandey S, Raza MS and Bhatta CP (2012). Prevalence and Antibiotic Sensitivity Pattern of MethicillinResistant Staphylococcus aureus involved in a hospital outbreak. J Clin Microbiol 37: 2858-2862.

Rajbhandari R, Manandhar SP and Shrestha J (2003). Comparative study of MRSA and its antibiotic susceptibility pattern in indoor and outdoor patients in Bir Hospital, Nepal. Nepalese J Microbiol 1: 62-65.
Raut S, Bajracharya K, Adhikari J, Pant SS and Adhikari B (2017). Prevalence of methicillin resistant Staphylococcus aureus in Lumbini Medical College and Teaching Hospital, Palpa, Western Nepal. BMC Res Notes 10:187.

Read AF and Woods RJ (2014). Antibiotic resistance management. Evol Med Public Health 1: 147.

Richmond VA (2000). Role of Penicillin Binding Protein Four (PBP4) in Vancomycin Susceptibility Among Clinical Vancomycin Intermediate Susceptible Staphylococcs aureus (VISA) Isolates. Antimicrob Agent Chemther 20: 40-75.

Rijal KR, Shrestha N, Pahari N, Shrestha B, Paudel B, Nepal A, Ghimire P and Rijal B (2008). Methicillin Resistant Staphylococcus aureus in patients visiting Western Regional Hospital, Pokhara. Journal of Institute of Medicine 30: 21-25.

Sit PS, Teh CSJ, Idris N, Sam IC, Omar SFS, Sulaiman H, Thong KL, Kamarulzaman A and Ponnampalavanar S (2017). Prevalence of methicillin-resistant Staphylococcus aureus (MRSA) infection and the molecular characteristics of MRSA bacteraemia over a two-year period in a tertiary teaching hospital in Malaysia. BMC Infect Dis 17: 274.

Spagnolo MA, Orlando P, Panatto D, Amicizia D, Perdelli F and Cristina LM (2014). Staphylococcus aureus with reduced susceptibility to vancomycin in healthcare settings. J Prev Med Hyg 55 (4): 137144 .

Spellberg B and Gilbert DN (2014). The future of antibiotics and resistance: a tribute to a career of leadership by John Bartlett. Clin Infect Dis 59(S2): S71-S75.

Surucuglu S, Gazi H, Kurutepe S, Ozkutak and Ozbakkaloglu B (2005). Bacteriology of surgical wound infection in Tertiary care Hospital in Turkey. Afr Med J 82(7): 3-6. 